Journal of Applied Fluid Mechanics, Vol. 10, Special Issue, pp. 33-39, 2017.

Selected papers from International Conference on Newer Engineering

Concepts and Technology, ICONNECT2K17, 2017

Available online at www.jafmonline.net, ISSN 1735-3572, EISSN 1735-3645

DOI: $10.36884 / \mathrm{jafm} .10$. SI.28268.

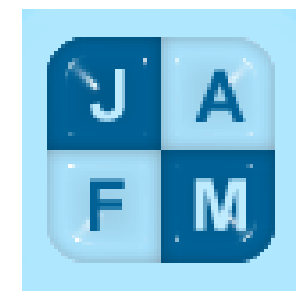

\title{
Computational Fluid Dynamic Analysis of Missile with Grid Fins
}

\author{
R. Srinivasan ${ }^{1 \dagger}$, V. Vijayan ${ }^{2}$ and K. Sridhar ${ }^{3}$ \\ ${ }^{1}$ Department of Mathematics, Kongunadu College of Engineering and Technology, Trichy, Tamilnadu, \\ 621215, India \\ ${ }^{2}$ Department of Mechanical Engineering, K. Ramakrishnan College of Technology, Trichy, Tamilnadu, \\ 621112, India \\ ${ }^{3}$ Department of Mechanical Engineering, Kongunadu College of Engineering and Technology, Trichy, \\ Tamilnadu, 621215, India \\ †Corresponding Author Email: srinivasanmaths@gmail.com
}

(Received February16, 2017; accepted July 30, 2017)

\begin{abstract}
This paper elucidates the outcomes of an experimental study validating a methodology for applying aerodynamic coefficients for a missile with grid fins. A grid fin is an unconventional lifting and control surface that consists of an outer frame supporting an inner grid of interesting planar surfaces of small chord. As a part of the experimentation, determinations were made based on the calculations at a match number of 0.2 and angle of attack $10^{\circ}$ for a missile with grid fins. The simulations were found to be efficacious as well as probable in determining the flow structure around the fin in the separated-flow region at the higher angles of attack. This was impeccably apparent in the successful calculation of nonlinear behavior viscous computational fluid dynamic simulations to calculate the flow field and for that fin, which indicated negative normal force at the higher angle of attack. The modeling of unconventional grid fin missile is done in CAD software named catia V5. The enmeshing of geometry is accomplished in a pre-processor named Gambit. In addition to that, the solving and the post processing are experimented in a solver called Fluent.
\end{abstract}

Keywords: Horizontal stabilizer; Pitch; Lift; Induced drag; Angle of attack.

\section{INTRODUCTION}

Abate, (G 1999 and 2000 ) said that, over the span of many years, a multitude number of research efforts are provoked and effected, by conducting inventive and resourceful explorations, in the form of experimental as well as theoretical, to improve the aerodynamics of flows in the unconventional grid fin missile. Burkhalter (1995) encountered these explorations, to a great extent, have steered to the formulation of empirical models, which created and customized a relationship with parameters like Mach number, pressure, aerodynamic coefficients to the overall performance of the missile. Aftosmis (1997 and 2000) revealed that there is an emerging demand of high performance and reliable unconventional grid fin missile, a much elemental approach viz. evolution of flow fields, aerodynamic coefficients and species concentration right the way through the domain of interest is indispensable. Burkhalter (1996) said that there has been an emergence in the development of procedures with the aid of computational systems for predicting flow fields in unconventional grid fin missile. John D (1995) and Robert D (2002) Prior to these computational methods, inquisitions have been ventured through experimental, theoretical and CFD approaches by many a number of investigators in this specific field of importance.

\subsection{Longitudinal Stabilization}

It consists of three types of plane.

\subsubsection{Tail Plane}

The tail plane is a small horizontal wing attached to the rear of an aircraft, forming part of the tail or empennage. It acts with the other horizontal surfaces to provide longitudinal (or "pitch") stability. Fixed horizontal stabilizers usually have hinged elevators attached to the rear of the surface to provide pitch control for the aircraft. Most large airliners and transports use an a combination of a stabilizer with an adjustable angle of incidence and independently moving elevators. The elevators are 
used by the pilot for rapid maneuvers and are controlled by the pilot's yoke. An adjustable stabilizer is adjusted to trim the aircraft's pitch to keep the elevators centered during normal flight. In aircraft with elevators, a trim tab on the trailing edge of the elevator is often used to move the elevator to alter the aircraft's. The stabilized speed is known as the trim speed, and the trim is used to set the desired speed without having to hold the elevator out of its trimmed or faired (trail) position. Other aircraft use an one-piece moving surface that serves the function of both a stabilizer and an elevator and is called a "stabilator" or "all moving tail plane". One example of a modern airliner with a stabilator used for flight control is the Lockheed L-1011 TriStar. Horizontal "Stabilators" are generally used for aircraft flying near or above the speed of sound because the formation of shock waves on the surface of the stabilizer tends to reduce the effectiveness of elevators. Stabilators may also use a trim tab to control the angle of the all moving stabilator surface, as on the Miles M.52. The North American F-86 Sabre initially used a fixed stabilizer and elevator with a trim tab, but later versions used a stabilator. For the aircraft to be stable, the horizontal stabilizer will often tend to exert a down force. This is particularly so at supersonic speeds: as the aircraft crosses the sound barrier its centre of lift moves aft, requiring greater down force from the tail plane in order to maintain trim. An aircraft wing deflects the airflow downwards and adds some turbulence to it. This "downwash" can meet the tail plane, affecting its performance.

It is empirical to document that grid fins have some advantages over conventional, planar fins. One of the advantages is the ability to sustain lift at higher angles of attack since grid fins do not have the same stall features of planar fins. Another merit is the movement of its hinge, because the hinges are subjected to only slight movement and so there becomes a possibility for the ability to reduce the size of control actuator systems. Since curvature of the grid fins had little influence on their performative operations, folding down the fins onto the missile body is a storage design advantage. The main detriment was found to be the higher degree of drag than that of planar fins; nevertheless techniques for curtailing drag by altering the grid fm frame crosssection shape were demonstrated (Miller and Washington 1994). These studies also exhibited that grid fins experience a loss in control effectiveness in the transonic regime due to flow choking in the individual cells.

\subsubsection{Canard fore Plane}

Canard fore planes, in themselves, tend to reduce static longitudinal stability. They are even used to intentionally destabilize some combat aircraft in order to make them more manoeuvrable (In this case, electronic flight control systems use the pitch control function of the canard fore plane to create artificial static and dynamic stability.) But in some cases the fore plane acts with the other horizontal surfaces to provide longitudinal (or "pitch") stability, in which case it acts as a stabilizer. Static pitch stability can be achieved in canard aircraft by making the lift slope of the fore plane less than the lift slope of the main wing "to achieve stability, the change in lift coefficient with angle of attack should be less than that for the main plane." For most airfoils, lift slope decreases with wing loading. Therefore, pitch stability may be achievable by assuring that the wing loading of the fore plane is greater than that of the main wing (that is, by moving the center of gravity forward).

\subsubsection{Tailless Plane}

Tailless aircraft lack a separate horizontal stabilizer. In a tailless aircraft, the horizontal stabilizing surface is part of the main wing. Longitudinal stability in tailless aircraft is generally derived from the design of the wing to move the Aerodynamic center behind the centre of gravity, for example by varying angle of incidence along sweepback and/or variation in airfoil.

\subsubsection{Directional Stabilization}

The vertical stabilizer or fin is fixed to the aircraft and normally supports the rudder. It is used to give directional (or yaw) stability to the aircraft. The vertical stabilizer often employs a small fillet at its forward base which helps to prevents a phenomenon called rudder lock or rudder reversal Rudder lock occurs when the force on a deflected rudder (in a steady sideslip) suddenly reverses as the vertical stabilizer stalls. This may leave the rudder stuck at full deflection with the pilot unable to recenter it. The filet is sometimes called a dorsal fin.

\subsubsection{Tailless Directional Stabilization}

The vertical stabilizer is by far the most common type of directional (or yaw) stabilizer, however, it is possible to design aircraft with no discrete vertical directional stabilizer. Hang gliders are a commonly seen example. The leading edge of a hang glider, viewed from above, is swept back in a V shape. As one side of the $\mathrm{V}$ rotates forward into the airstream, the drag on the forward $1 / 2$ wing increases while the drag on other $1 / 2$ wing decreases. This tends to rotate the whole wing back so that it is facing straight into the airflow. (Similar to dihedral roll stabilization.)

\subsubsection{V - Tail}

A V-tail is a 2-fin combined vertical-horizontal stabilizer. V-tails combine the functions of both horizontal and vertical stabilizers, and a rudder and elevator, into a slightly simpler mechanical design. A V-tail has two stabilizer fins mounted at 45 degree angles to the horizontal and vertical planes. Each has a moving surface known as a "ruddervator".

\section{Modeling Process}

\subsection{Design Characteristics}

Conventional "planar" control fins are designed with the shape representing the miniature wings. In contradiction to that, grid fins are a lattice of smaller aerodynamic surfaces arranged within a box. It is 
factual to document that their distinctive appearance has sometimes subjected them to be compared on par with potato mashers or waffle irons.

Grid fins consists of unique mechanism that they could be folded against the body of missile more feasibly than planar fins, consenting to more compact storage of the weapon; this mechanism is of immense significance for craft which store weapons in internal bays, such as stealth aircraft. Instantaneously after the release, the fins are swivelled into place for operation as control surfaces.

In the case of MAOB, grid fins let the weapon to get fixed inside a C-130 cargo bay for deployment while the craft is on flight. Grid fins comprise of a diminutive "chord" (the distance between leading and trailing edge of a surface) than planar fins, as they are a group of efficiently shorter fins soared parallel to one another. Their reduced chord condenses the amount of torque exerted on steering mechanism by high speed airflow, giving the probability for the use of smaller fin actuators, and a smaller tail assembly overall.

Their petite chord also lets them to be accumulated to the degree of less prone to stall at high angles of attack, allowing for tighter turns. Grid fins perform deftly at subsonic and supersonic speeds, but scantily at transonic speeds; the flow causes a normal shock wave to form within the lattice, effecting in the event that much of the airflow pass completely around the fin instead of through it and generates significant wave drag. However, at high Mach numbers, grid fins flow fully supersonic and can offer lower drag and greater manoeuvrability than planar fins.

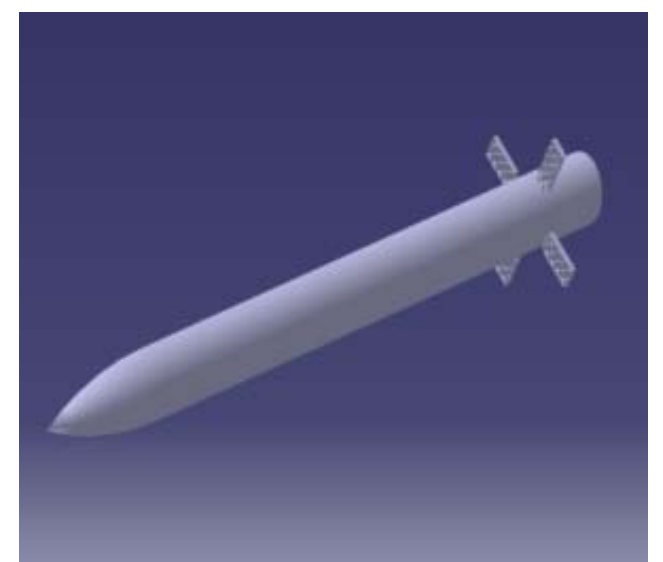

Fig. 1. 3D view of the missile.

\subsection{Description of the Geometry}

Missile has been designed by using catia v5 and the execution of grid fins with the missile is devised and effected warily. Planar fans are entirely dissimilar to grid fins, which include diverse lattice arrangements which are classified into following categories.
i) Baseline lattice grid fin
ii) Coarse lattice grid fin

\section{iii) AFIT lattice grid fin}

It is weened that the AFIT design would preferably be better than the other lattice grid fins, because this layout enhances for doubling the area of the cells in the main body of the fin while enduring a similar shape.

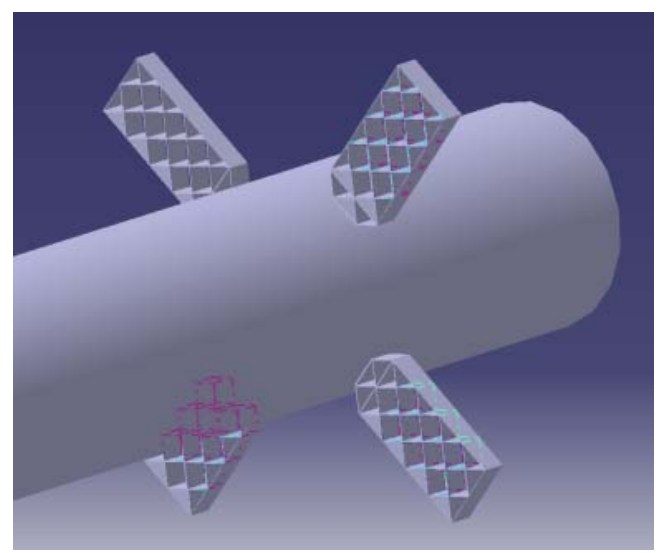

Fig. 2. Enlarged view of fins on missile.

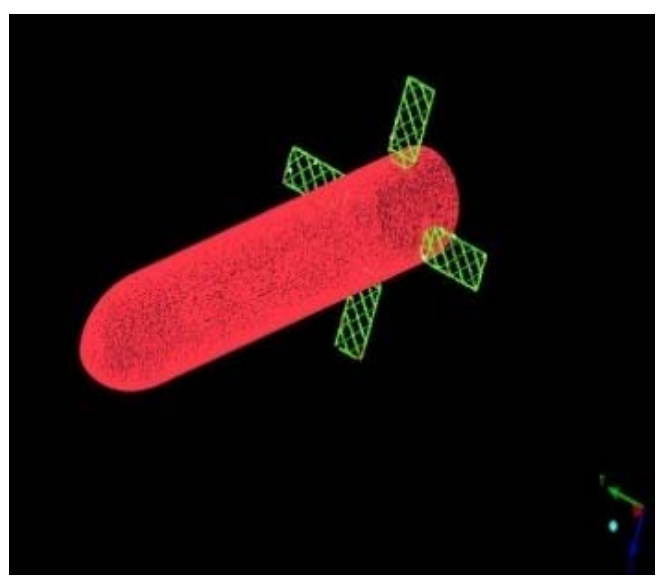

Fig. 3.Triangle mesh on missile surface.

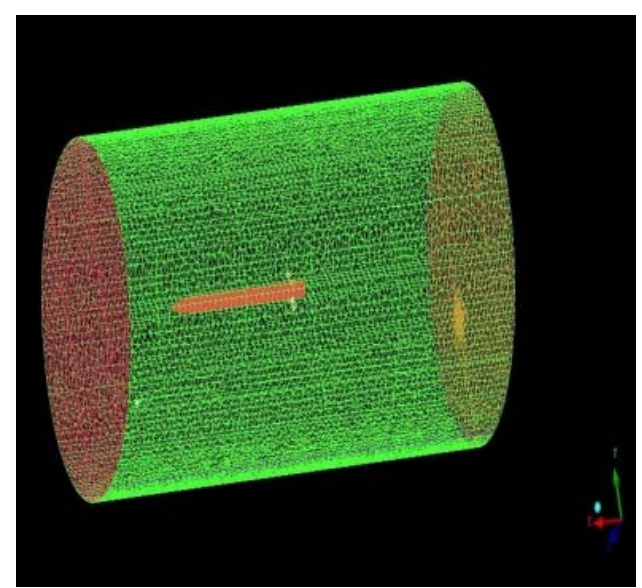

Fig. 4. Surface mesh on domain.

\subsection{Description of Meshing}

The missile geometry created in Catia V5 is 
imported into ICEM CFD for meshing by using suitable file acceptance format. As an initiative process, heed had been taken to create surface mesh on missile and fins. Further, the triangle elements have been exerted for this surface meshing and then the whole domain is meshed wielding tetrahedral cells. The quality of meshing found to be competent while it had been put to test by checking criteria like quality, aspect, ratio etc. In addition to that, a three dimensional unstructured tetra grid (mesh) has been germinated employing the tetra meshing feature of ICEM CFD. The mesh size is 2 million cells; the grid is refined in the near wall regions by means of prism cells. The three dimensional view of the full grid in ICEM CFD is shown in the figure below.

Thus the tetrahedral mesh for missile geometry is created using ICEM CFD. The tetrahedral ingredients are wielded for a much feasible meshing as it is unstructured and entails a lesser amount of time to get accomplished. Total elements: 17711046 and Total nodes: 287557. Thus both the elements and nodes perpetuate nearly 2 million cells. Then the mesh file is transmitted to suitable solver.

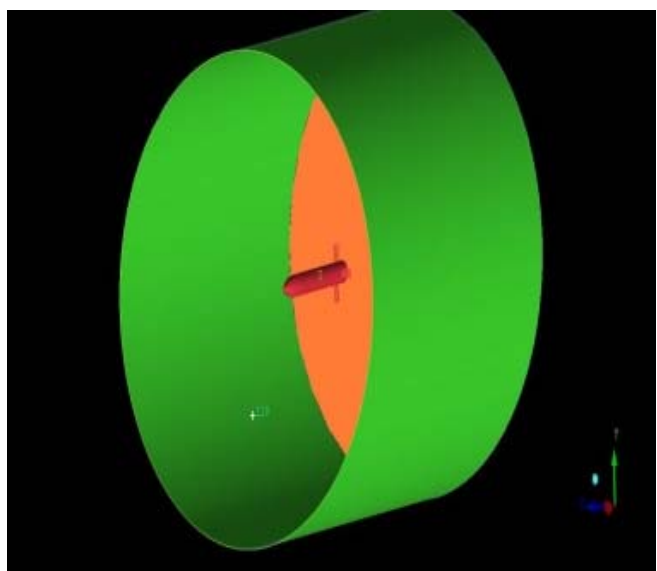

Fig. 5. 3D view of missile with domain in ICEM CFD.

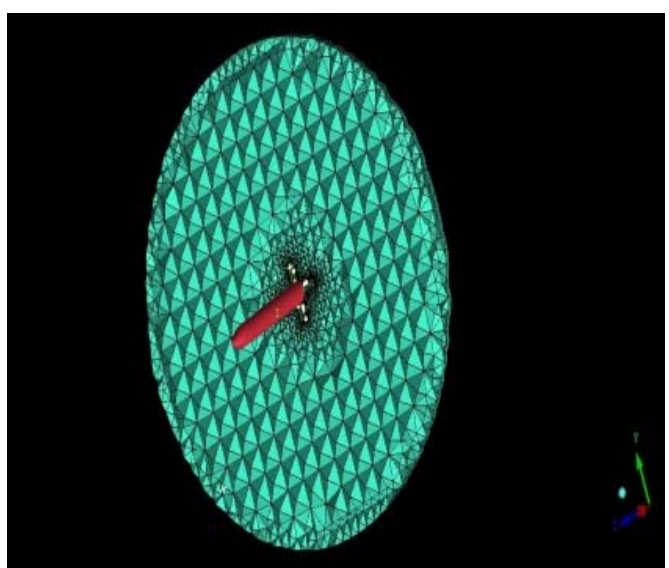

Fig. 6.Tetrahedral mesh cut plane on $x$-axis on domain.

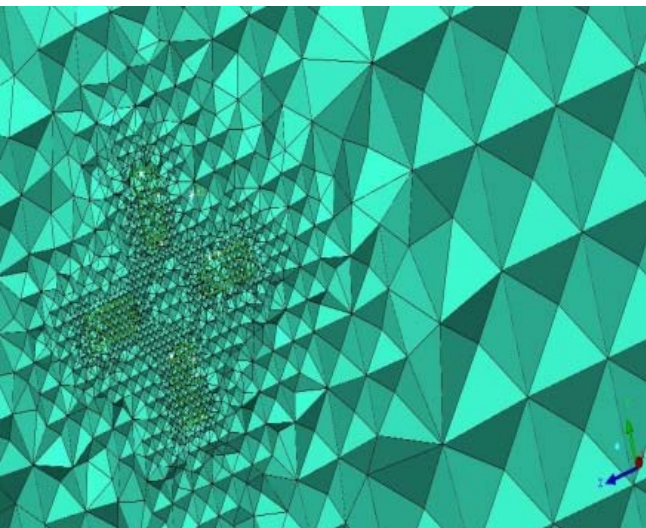

Fig. 7. Enlarged view of tetrahedral mesh cut plane on fins.

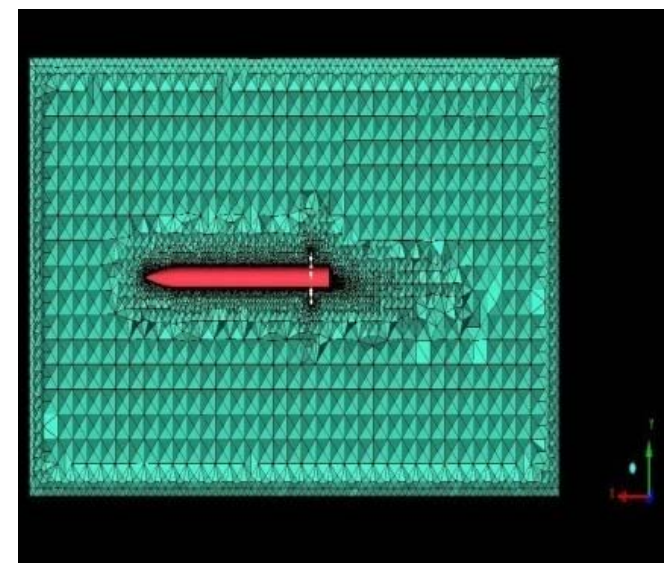

Fig. 8. Tetrahedral mesh cut plane on $\mathrm{z}$-axis on domain.

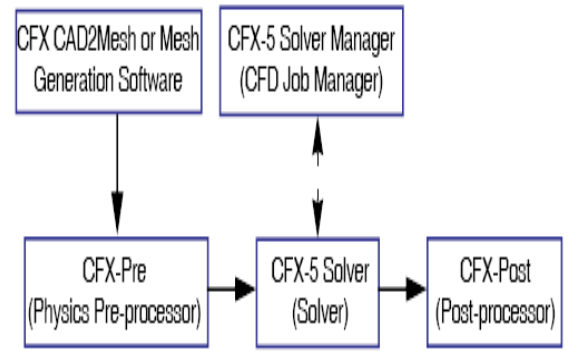

Fig. 9. The Structure of CFX.

Thus the tetrahedral mesh for missile geometry is created using ICEM CFD. The tetrahedral ingredients are wielded for a much feasible meshing as it is unstructured and entails a lesser amount of time to get accomplished. Total elements: 17711046 and Total nodes: 287557. Thus both the elements and nodes perpetuate nearly 2 million cells. Then the mesh file is transmitted to suitable solver.

\subsection{Description of Solver}

CFX is a general purpose Computational Fluid Dynamics (CFD) code, integrating an advanced solver with potent pre and post-processing competences. The next-generation physics preprocessor, CFX-pre consents multifarious meshes to 
R. Srinivasan et al. / JAFM, Vol. 10, Special Issue, pp. 33-39, 2017.
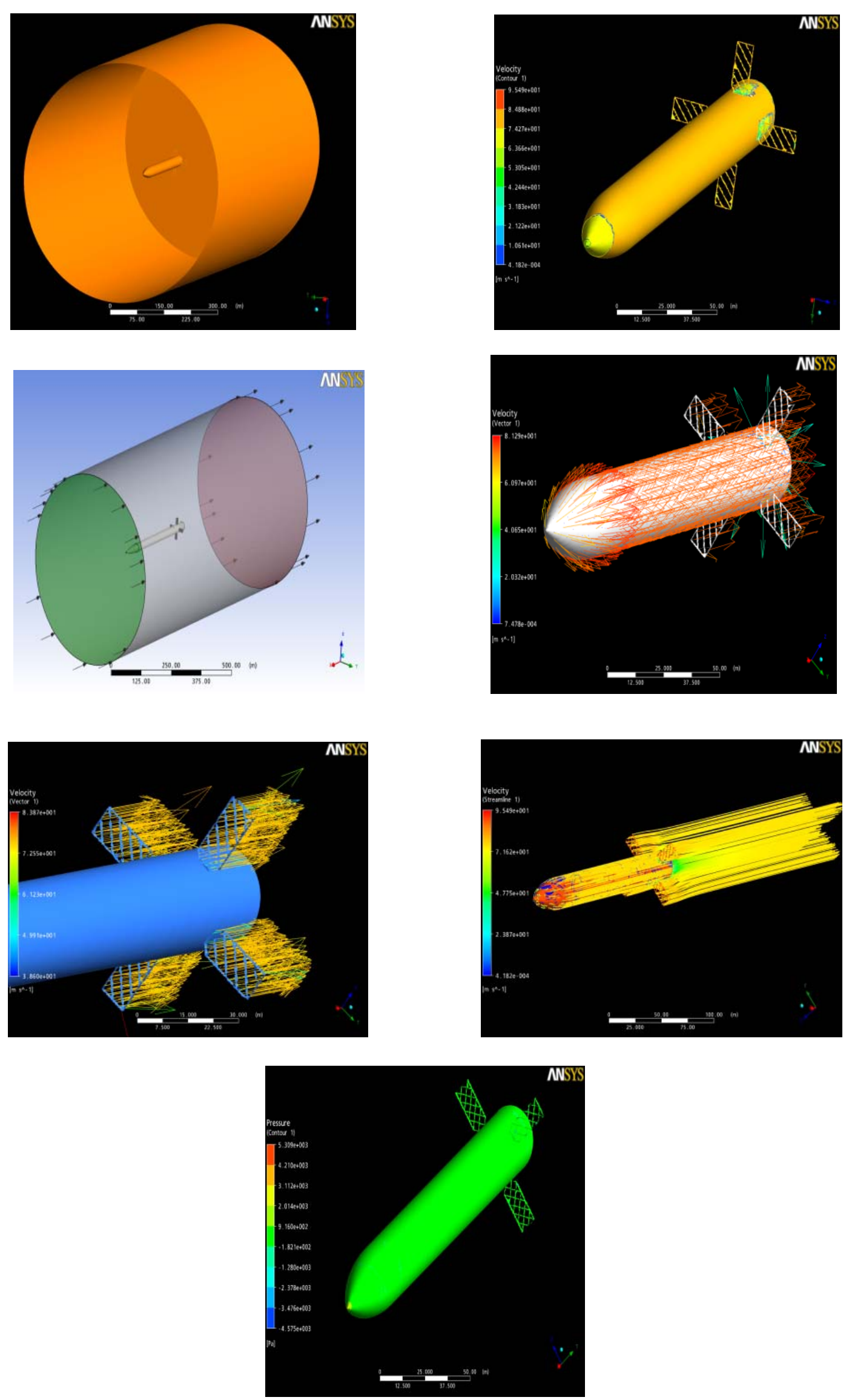

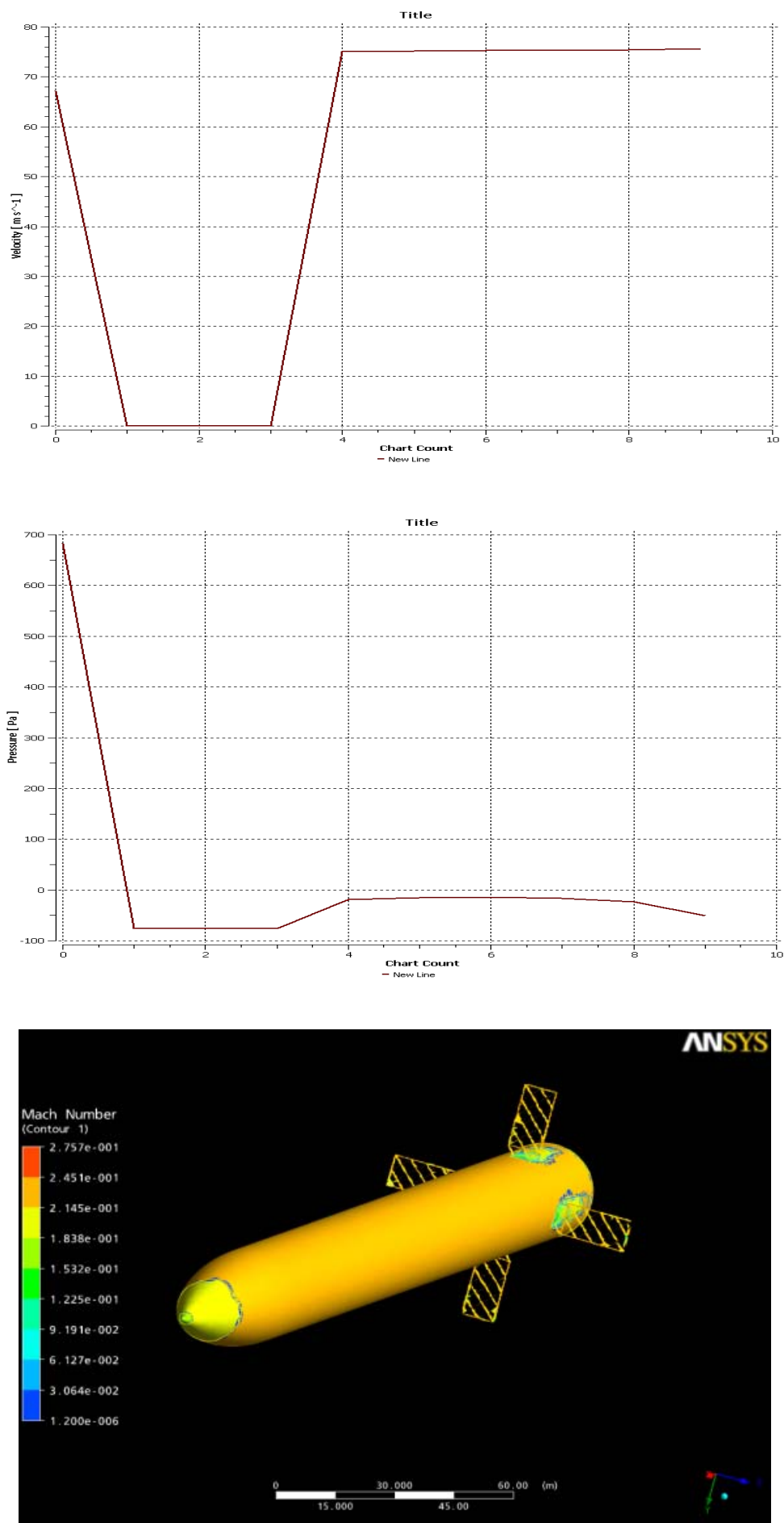

Fig. 10. Complex geometric view.

be imported, letting each fragment of complex geometries to use the most appropriate mesh.

CFX includes the following features:

(a) An advanced coupled solver which is both reliable and robust.

(b) Full integration of problem definition, analysis and results presentation. (c) An intuitive and interactive setup process, using menus and advanced graphics

(d) Detailed online help

\section{CONCLUSION}

The velocity contour indicates colour which contrasts from region to region. The maximum 
velocity acceleration due to the existence of fins and the body dimensions were indicated in the leading verge (front side-yellow colour) and the ones which has maximum velocity decelerations were indicated in trailing verge backside - blue colour) as shown. Stream plot is drawn over the missile with grid fin to view the flow visualisation for zero angle of attack. The avenge match number over missile is 0.21 (i.e) subsonic as per our supposition. Thus, the flow over missile is found to be adequate.

\section{REFERENCES}

Abate, G., R. P. Duckerschein and G. Winchenbach (1999, November). "Free-Flight Testing of Missiles with Grid Fins.” Proceedings of the 50th Aeroballistic Range Association Meeting, Pleasanton, CA.

Abate, G., R. P. Duckerschein and W. Hathaway (2000). Subsonic/Transonic Free-Flight Tests of a Generic Missile with Grid Fins. AIAA Paper 2000-0937.
Aftosmis, M. J. (1997) Solution Adaptive Cartesian Grid Methods for Aerodynamic Flows with Complex Geometries. Computational Fluid Dynamics VKI Lectures Series 1997-05, von Karman Institute for Fluid Dynamics, Belgium.

Aftosmis, M. J. (2000). Personal communication. NASA Ames Research Center, Moffett Field, CA.

Burkhalter, J. E. (1996). Grid Fins for Missile Applications in Supersonic Flow. AIAA Paper 96-0194.

Burkhalter, J. E., R. J. Hartfield and T. M. Leleux (1995). Nonlinear Aerodynamic Analysis of Grid Fin Configurations. J. ofAircraft 32(3), 547-554.

John, D. and J. Anderson (1995). Computational Fluid Dynamics the basics with applications, Mc Graw- Hill,Inc.

Robert, D. Z. and O. Biblarz (2002). Fundamentals of Gas Dynamics John Wiley and sons. Inc. 